\title{
The self-thinning rule and plant population modelling with resource constraints
}

Dimetre Triadis ${ }^{1} \quad$ Robert McKibbin ${ }^{2} \quad$ Boris Baeumer $^{3}$

(Received 17 March 2018; revised 14 May 2018)

\begin{abstract}
The Japan Agency for Marine-Earth Science and Technology contributed this challenge to the 2016 Mathematics-in-Industry New Zealand Study Group Workshop. It concerned implications of the self-thinning rule for modelling plant population characteristics via a partial differential equation governing the temporal evolution of the density distribution of plants of a particular size. The self-thinning rule is empirically observed for crowded populations under constrained resources. We investigate the theoretical consequences of a resource constraint on the partial differential equation of interest, and through numerical experiments reveal a surprisingly strong link between imposition of the resource constraint, and populations that evolve according to the self-thinning rule. The result is a simple condition between growth and mortality functions that implies self-thinning behaviour, and motivates further mathematical investigation.
\end{abstract}

DOI:10.21914/anziamj.v58i0.12998, C) Austral. Mathematical Soc. 2018. Published 2018-05-27, as part of the Proceedings of the 2016 Mathematics and Statistics in Industry NZ Study Group. ISSN 1445-8810. (Print two pages per sheet of paper.) Copies of this article must not be made otherwise available on the internet; instead link directly to the DOI for this article. 
Subject class: 35Q92

Keywords: self-thinning; PDE; population modelling

\section{Contents}

1 Introduction

M366

2 Resource constraints and the self-thinning rule

M369

3 Numerical experiments

M371

3.1 Unimodal initial condition . . . . . . . . . . . . . . M371

3.2 Bimodal initial condition . . . . . . . . . . . . . M372

3.3 Effects of increased diffusion . . . . . . . . . . . M374

4 Conclusion

M374

\section{Introduction}

When plant populations become crowded and resources are almost fully utiised, the mortality of individuals is largely a consequence of competition for scarce resources. Such competition-induced mortality is referred to as selfthinning, as opposed to thinning that results from external factors. The selfthinning rule $[6,10]$ is a relationship between the average size of individuals $\bar{x}$ and the spatial population density $\rho$, often measured as the number of individuals per unit area. We are not directly concerned with the spatial arrangement of individual plants, but assume some homogenous population density distribution $f(t, x)$ of individuals of various sizes $A \leqslant x \leqslant B$, per unit area (or for the area of interest) at some point in time:

$$
\rho(t)=\int_{A}^{B} f(t, x) d x, \quad \bar{x}(t)=\frac{1}{\rho(t)} \int_{A}^{B} x f(t, x) d x .
$$


For some constant $\mathrm{K}$, the self-thinning rule is

$$
\bar{x}=K \rho^{-\mathrm{c}}, \quad \mathrm{c} \simeq 3 / 2 .
$$

It is not a rule that explicitly governs the evolution of $\rho(t)$ and $\bar{x}(t)$ : it merely specifies the boundary of possible $(\rho, \bar{x})$ combinations. Given a population density $\rho$, values of $\bar{x}$ less that that specified by the self-thinning rule are possible, and imply an incomplete use of available resources. Populations of this type that are not crowded tend to evolve towards the boundary dictated by the self-thinning rule as time progresses and they grow to use more of the available resources. When the population does become crowded, the self-thinning rule dictates that if the average size of individuals is to increase (implying an increase in biomass density), then this increase must come at the expense of the population density so that the boundary (1) is not crossed.

Section 2 discusses some simple theoretical arguments that shed light on why (1) might apply in the presence of resource constraints. However, the self-thinning rule is primarily an empirical observation supported by a body of experimental biological data [4]. It is usually qualified as applying to populations that are not just crowded, but also monospecific, or of evenage $[8,7]$, although some studies considered generalisations that apply across species mixtures [9]. The self-thinning rule is also observed in, and applied to, sessile and mobile animal populations [3,5]. While the universality of the value $c=3 / 2$ has been the subject of debate in the plant literature, that is not our concern in the present study, where we retain the freedom to alter $\mathbf{c}$.

A standard model for the evolution of the population density distribution $f(x, t)$ is that used by Hara [2]:

$$
\frac{\partial f(t, x)}{\partial t}=\frac{1}{2} \frac{\partial^{2}}{\partial x^{2}}[D(t, x) f(t, x)]-\frac{\partial}{\partial x}[G(t, x) f(t, x)]-M(t, x) f(t, x) .
$$

Here $G(t, x)$ and $D(t, x)$ are, respectively, the mean and variance of the growth rate of $x$; and $M(t, x)$ is a mortality rate.

The 2016 Mathematics-in-Industry New Zealand Study Group Workshop (MINZ) team was asked to investigate relationships between the functions G, D, 
and $\mathbf{M}$ of PDE (2) so that the temporal evolution of the plant population $\mathbf{f}(\boldsymbol{t}, \boldsymbol{x})$ obeys the self-thinning rule (1).

From the Japan Agency for Marine-Earth Science and Technology (JAMSTEC) problem description, values $A$ and $B$ can be considered isolated: we are not concerned with recruitment of individuals via reproduction and begin with an established population of individuals with $x>A$ such that $f(t, A)=0$. We expect that growth of young individuals dominates any diffusive effects governed by $\mathrm{D}(t, x)$ that might tend to decrease plant size. As such the flux

$$
J(t, x)=G(t, x) f(t, x)-\frac{1}{2} \frac{\partial}{\partial x}[D(t, x) f(t, x)],
$$

is assumed to be essentially zero at $x=A$. Setting $B$ large enough, we also assume that $f(t, B)=0$ with negligible flux at $x=B$. The isolation of $x=B$ is either due to a restricted time frame, or at longer times scales due to the mean growth rate $G$ being suppressed for large $x$, as organisms reach the limit of their natural size.

There is some conflict between the condition that self-thinning populations be monospecific or even-aged and the PDE (2) due to the diffusion term incorporating $\mathrm{D}(\mathrm{t}, \mathrm{x})$. A group of organisms which start out very similar to one another will soon exhibit a range of different sizes due to natural variation of their growth rates, and this effect may be significant for high values of $\mathrm{D}(\mathrm{t}, \mathrm{x})$. The numerical experiments of Section 3 show behaviour consistent with the self-thinning rule arising as a result of imposition of a resource constraint, even when $\mathrm{D}(\mathrm{t}, \mathrm{x})$ has a significant effect or the population distribution is initially bimodal. 


\section{Resource constraints and the self-thinning rule}

Limited understanding of the self-thinning rule is gained by considering some finite resource, such as a two-dimensional substrate, that scales in a different manner to organism size $x$ as a population of identical organisms grow. Let us assume a characteristic length $l$ for each plant so that the resource $r$ taken by each plant, and the size $x$ of each plant, are

$$
r=k_{r} l^{2}, \quad x=k_{x} l^{3},
$$

for some constants $k_{r}$ and $k_{x}$. Assuming a constant total resource $R=\rho r$, we eliminate $l$ to obtain the self-thinning rule

$$
x=K \rho^{-3 / 2}, \quad K=\frac{k_{x} R^{3 / 2}}{k_{r}^{3 / 2}} .
$$

The above is equivalent to

$$
\mathrm{R}=\frac{\mathrm{k}_{\mathrm{r}}}{\mathrm{k}_{\mathrm{x}}^{2 / 3}} \rho x^{2 / 3} .
$$

Should the resource $r$ or the size $x$ exhibit a different scaling to those shown in (4), versions of the self-thinning rule with $c \neq 3 / 2$ are easily derived as above.

As pointed out by White [8] and others, a key problem with the simple argument above is that plants and other organisms may not grow isometrically, so that $k_{r}$ and $k_{x}$ may not remain constant as $l$ varies. Various attempts to derive self-thinning rules for particular species based on more biologically realistic scaling arguments have been made, and often produce values of $c$ from (1) close to $3 / 2$ [1].

As mentioned in the Introduction, these simple scaling arguments for identical organisms are somewhat in conflict with the diffusive nature of PDE (2). 
One might consider two sub-populations of characteristic lengths $l_{1}$ and $l_{2}$, but this does not lead to an equation like (5) without adding some strong mathematical assumptions.

For a population exhibiting a continuous distribution of sizes, we assume that organism resource use is given by $r(x)$ so that equation (6) is generalised to

$$
R=\int_{A}^{B} r(x) f(t, x) d x,
$$

where for our purposes the organism resource use of primary interest is $r(x)=x^{1 / c}$.

The self-thinning rule is a consequence of resource constraints under crowded conditions. Rather than approach the self-thinning rule directly, there is a more natural mathematical path to considering the compatibility of the above resource constraint with the differential PDE (2). Differentiating the equation for $\mathrm{R}$ above with respect to time,

$$
0=\int_{A}^{B} r(x) f_{t}(t, x) d x .
$$

Substituting the right-hand side of PDE (2), and carrying out integration-byparts twice then produces

$$
\begin{aligned}
0= & {\left[\left\{-r(x) G-\frac{1}{2}\left[r(x) D_{x}-r^{\prime}(x) D\right]\right\} f(t, x)+\frac{1}{2} r(x) D f_{x}(t, x)\right]_{A}^{B} } \\
& +\int_{A}^{B}\left[r^{\prime}(x) G-r(x) M+\frac{1}{2} r^{\prime \prime}(x) D\right] f(t, x) d x .
\end{aligned}
$$

The boundary isolation previously discussed enables us to neglect the first term in square brackets above. The derivation then implies that the PDE (2) is compatible with the resource constraint (7) if the following condition is satisfied

$$
M(t, x)=\frac{r^{\prime}(x)}{r(x)} G(t, x)+\frac{r^{\prime \prime}(x)}{2 r(x)} D(t, x) .
$$


Does the above condition on $\mathrm{M}, \mathrm{G}$ and $\mathrm{D}$ also produce self-thinning type behaviour in the governing PDE (2)? We explore this question computationally in the following section.

\section{$3 \quad$ Numerical experiments}

To test the conjecture above concerning the link between equation (9) and the self-thinning rule we solved the PDE (2) numerically over the domain $1 \leqslant x \leqslant 100,0 \leqslant t \leqslant 1$. The spatial domain was discretised into 500 nodes, and the resulting system of ordinary differential equations was solved using Matlab's ode45() function with a temporal grid spacing of 0.01 .

\subsection{Unimodal initial condition}

We start by considering an initial condition

$$
f(0, x)=\sqrt{\frac{20}{\pi}} \exp \left[-20(x-20)^{2}\right],
$$

representing an population of individuals at $t=0$ of approximate size $x=20$.

Figure 1 shows both the evolution of $f(t, x)$ and the resulting changes in $\rho$ and $\bar{x}$ as time progresses. Here the mean growth rate was simply $\mathrm{G}=45$, with $\mathrm{D}=0.02 \chi$, and $M$ was determined according to equation (9) with the standard values $c=3 / 2$ and $r=x^{1 / c}$ adopted. The reference line labelled on the second panel of Figure 1 shows the self-thinning rule (1) with $\mathbf{c}=3 / 2$, and $\mathrm{K}$ chosen to intersect the first numerical result at $\mathrm{t}=0$. The numerical results are in excellent agreement with the predictions of the self-thinning rule despite equation (9) having been derived only by considering the resource constraint (7).

Numerical results were obtained for all 15 pairwise combinations of the G and $\mathrm{D}$ functions shown in Table 1 . Whenever the initial condition was given 
Figure 1: Numerical solution of PDE (2) with unimodal initial condition (10), $\mathrm{G}=45, \mathrm{D}=0.001 \chi^{2}$, and $\mathrm{M}$ determined according to equation (9).
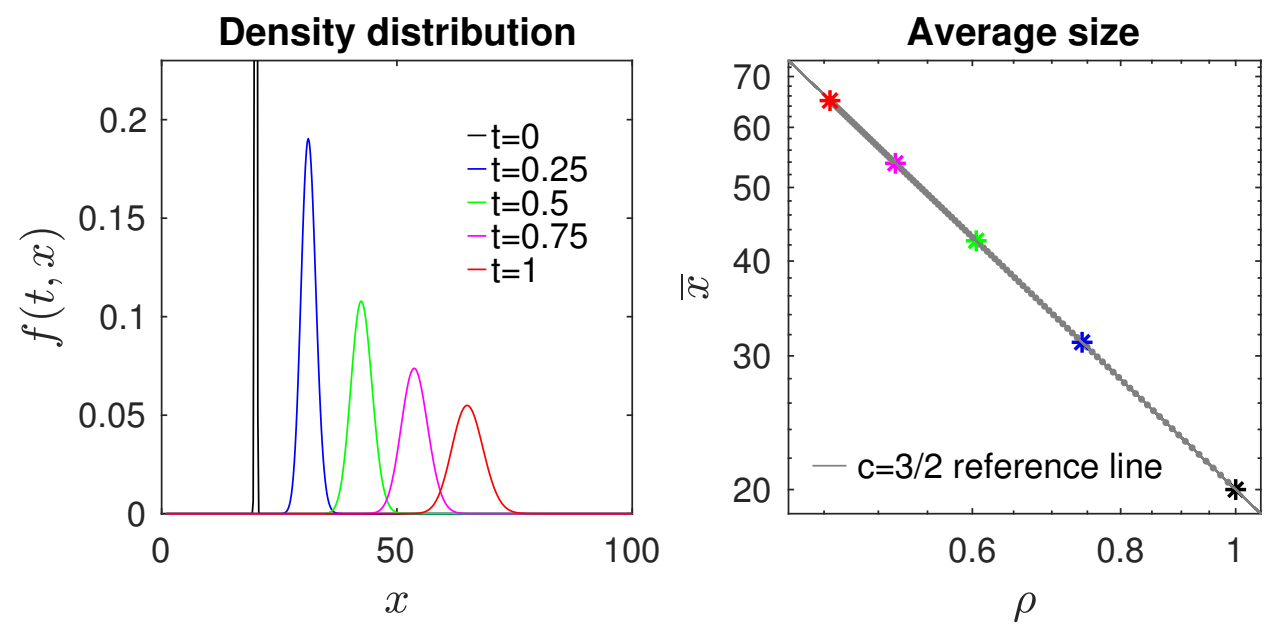

according to (10), we observed excellent agreement with the self-thinning rule, similar to that shown graphically in Figure 1.

Moreover, for all 15 cases, the numerical results appeared in excellent agreement with the self-thinning rule even when $M$ was determined independently of D using

$$
M(t, x)=\frac{r^{\prime}(x)}{r(x)} G(t, x) .
$$

\subsection{Bimodal initial condition}

The results described above exhibit quite precise self-thinning behaviour despite the diffusive effects in PDE (2) tending to produce organisms with greater size variation as time progresses. We conduct a related, but more 
Table 1: Various $G$ and D test functions used in numerical experiments.

\begin{tabular}{cc}
\hline G functions & D functions \\
\hline 45 & 0.2 \\
$0.9 x$ & $0.001 \chi^{2}$ \\
$100-x$ & $0.2 \mathrm{G}$ \\
$0.4 \times(10-\sqrt{x})$ & \\
$100(1.1+\sin (x))$ & \\
\hline
\end{tabular}

Figure 2: Numerical solution of PDE (2) with bimodal initial condition (12), $\mathrm{G}=100-x, \mathrm{D}=0.2 \mathrm{G}$, and $\mathrm{M}$ determined according to equation (11).
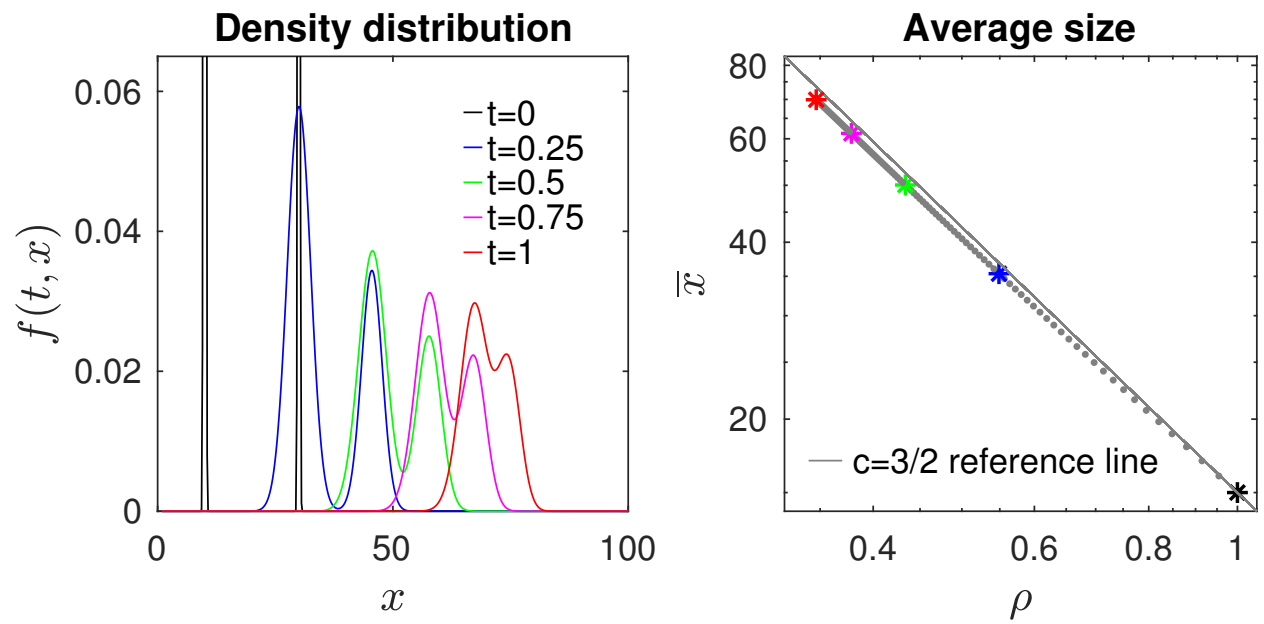

severe test of our system by adopting the initial condition:

$$
f(0, x)=\frac{3}{2} \sqrt{\frac{5}{\pi}} \exp \left[-20(x-10)^{2}\right]+\frac{1}{2} \sqrt{\frac{5}{\pi}} \exp \left[-20(x-30)^{2}\right],
$$

describing two subpopulations of distinct sizes 10 and 30 at time $t=0$.

Working through the 15 cases of Table 1 with this new initial condition, it is observed that the self-thinning rule is still approximately satisfied, although 
not as accurately as illustrated in Figure 1. Typical results are shown in Figure 2, for $\mathrm{G}=100-x, \mathrm{D}=0.2 \mathrm{G}$, and $\mathrm{M}$ determined according to the simpler equation (11). The relationship between (9) or (11) and the self-thinning rule hence appears to be relatively robust when tested with a population that does not consist of similar individuals. This is despite the self-thinning rule usually being presented as valid only for populations of similar organisms.

\subsection{Effects of increased diffusion}

The calculations described show little observable difference between imposing the full condition (9), or condition (11) where the term involving D has been neglected. To investigate further we conduct calculations with relatively slow growth rates and higher values of $\mathrm{D}$. The calculations of Figure 3 use the bimodal initial condition

$$
f(0, x)=\sqrt{\frac{5}{\pi}} \exp \left[-20(x-30)^{2}\right]+\sqrt{\frac{5}{\pi}} \exp \left[-20(x-50)^{2}\right],
$$

with $G=(100-x) / 5$ and $D=15 G$. The upper plots show $M$ determined according to (9) with the term involving D included, whereas the lower plots define $M$ according to (11). We observe that the final term of (9) does have an influence in this case, and acts to generate a more accurate reproduction of the self-thinning rule. The effect illustrated in Figure 3 appears to be typical, but not universal for calculations where $\mathrm{D}$ is exaggerated.

\section{Conclusion}

Rather than attempt to impose the self-thinning rule directly on the PDE (2) of interest, we found it theoretically more convenient to seek PDEs (2) that satisfy a resource constraint (7). The self-thinning rule has been empirically observed 
Figure 3: Numerical solution of PDE (2) with bimodal initial condition (13), $\mathrm{G}=(100-\chi) / 5, \mathrm{D}=15 \mathrm{G} . \mathrm{M}$ is determined according to $(9)$ in the upper plots, and according to (11) in the lower plots.
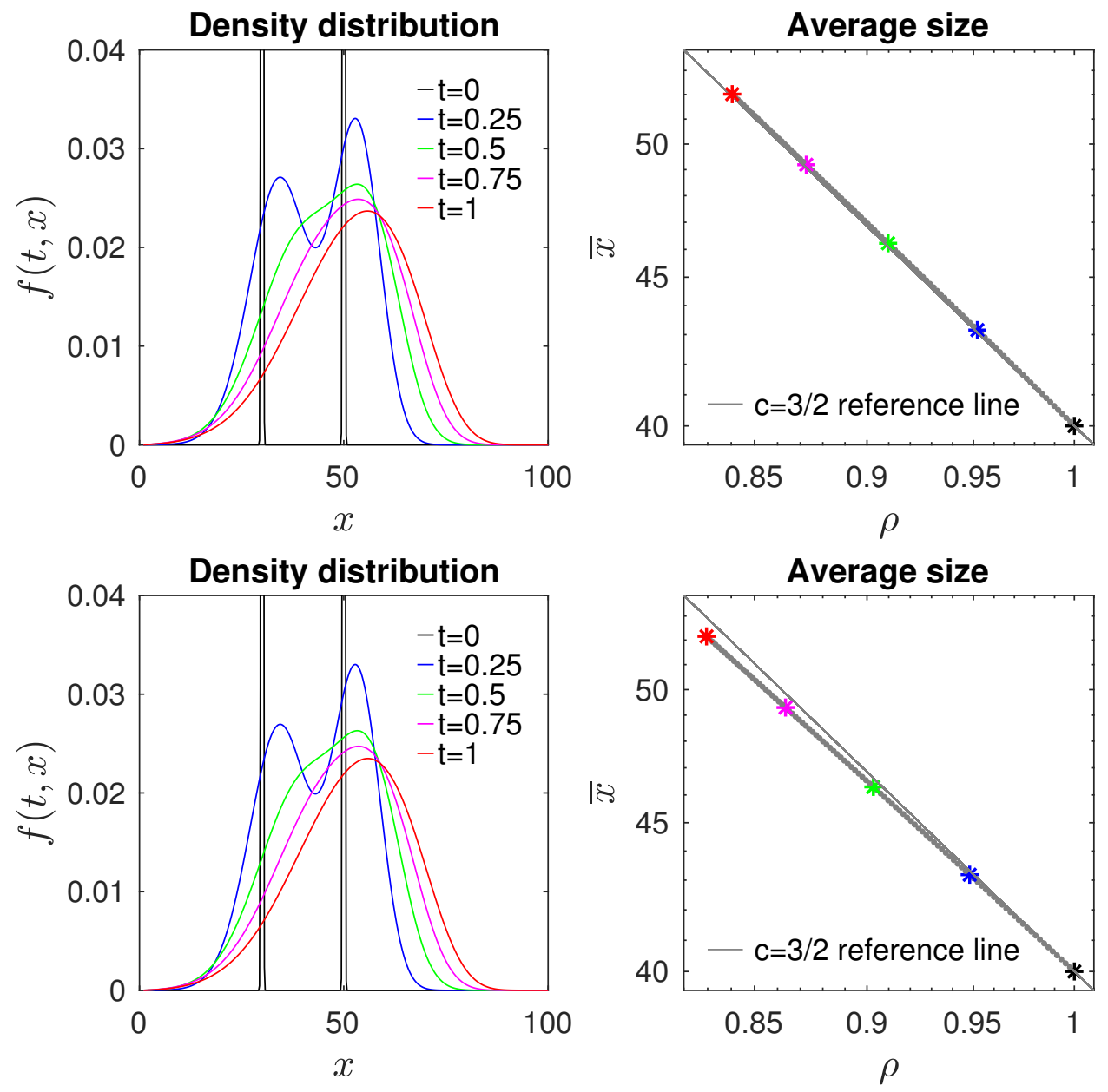
in biological populations of similar individuals under crowded conditions where resources are scarce. However, we are aware of no clear mathematical link between equations (1) and (7) which embody the self-thinning rule and a resource constraint respectively.

Had the numerical experiments of Section 3 failed to exhibit behaviour consistent with the self-thinning rule, it would not have been surprising. Instead, we appear to observe a strong link between the consequences of (7) and the self-thinning rule. This link is surprisingly robust when considering populations that are not made up of similar individuals, but at times consist of two distinct organism sizes. Diffusive effects tend to produce populations with a variety of sizes, but the link between the self-thinning rule and the resource constraint (7) appears quite insensitive to the term D in PDE (2), so much so that neglecting the final term of (11) appears to have a negligible effect in most of the cases that we considered. The link also appears to be robust with respect to the particular value of $\mathrm{c}$ used in equation (1), provided that we adopt a matching $r(x)=x^{1 / c}$ term to define our resource constraint (7).

Acknowledgements We express our gratitude to the organisers of the MINZ-2016 Study Group Workshop, and especially to Dr Hirofumi Sakuma of JAMSTEC for preparing such an interesting workshop problem.

\section{References}

[1] Brian J. Enquist, James H. Brown, and Geoffrey B. West. "Allometric scaling of plant energetics and population density". In: Nature 395 (1998), pp. 163-165. DOI: 10.1038/25977 (cit. on p. M369).

[2] Toshihiko Hara. "A stochastic model and the moment dynamics of the growth and size distribution in plant populations". In: Journal of Theoretical Biology 102.2 (1984), pp. 173-190. DOI: 10.1016/S0022-5193(84)80002-8 (cit. on p. M367). 
[3] R. N. Hughes and C. L. Griffiths. "Self-Thinning in Barnacles and Mussels: The Geometry of Packing". In: The American Naturalist 132.4 (1988), pp. 484-491. DOI: 10.1086/284866 (cit. on p. M367).

[4] W. M. Lonsdale. "The Self-Thinning Rule: Dead or Alive?" In: Ecology 71.4 (1990), pp. 1373-1388. DOI: 10.2307/1938275 (cit. on p. M367).

[5] Stefan O. Steingrimsson and James W. A. Grant. "Allometry of territory size and metabolic rate as predictors of self-thinning in young-of-the-year Atlantic salmon". In: Journal of Animal Ecology 68 (1999), pp. 17-26. DOI: 10.1046/j.1365-2656.1999.00261.x (cit. on p. M367).

[6] Y. Tadaki and T. Shidei. "Studies on the competition of forest trees. II. The thinning experiment on small model stand of Sugi (Cryptomeria japonica) seedlings." In: Nippon Rin Gakkaishi 41 (1959), pp. 341-349. DOI: 10.11519/jjfs1953.41.9_341 (cit. on p. M366).

[7] Mark Westoby. "The self-thinning rule". In: Advances in Ecological Research 14 (1984), pp. 167-225. DOI: 10.1016/S0065-2504(08)60171-3 (cit. on p. M367).

[8] James White. "The allometric interpretation of the self-thinning rule". In: Journal of Theoretical Biology 89 (1981), pp. 175-500. DOI: 10.1016/0022-5193(81)90363-5 (cit. on pp. M367, M369).

[9] James White. "The thinning rule and its application to mixtures of plant populations". In: Studies on Plant Demography. Ed. by James White. New York: Academic Press, 1985, pp. 291-309 (cit. on p. M367).

[10] K. Yoda et al. "Self-thinning in overcrowded pure stands under cultivated and natural conditions". In: J. Znst. Polytech. Osaka City Univ. Ser. D. 14 (1963), pp. 107-129. URL: https://ci.nii.ac.jp/naid/10004057399/en/(cit. on p. M366). 


\section{Author addresses}

1. Dimetre Triadis, Institute of Mathematics for Industry, Kyushu University, JAPAN.

mailto:D.Triadis@latrobe.edu.au orcid:0000-0002-1864-8396

2. Robert McKibbin, Institute of Natural and Mathematical Sciences, Massey University, NEW ZEALAND. mailto:R.McKibbin@massey.ac.nz

3. Boris Baeumer, Department of Mathematics and Statistics, University of Otago, NEw ZEALAND.

mailto:bbaeumer@maths . otago .ac.nz orcid:0000-0003-3841-1094 